\title{
Choreographing lived experience: dance, feelings and the storytelling body
}

\author{
Karin Eli, ${ }^{1}$ Rosie Kay ${ }^{1,2}$
}

- Additional material is published online only. To view please visit the journal online (http://dx.doi.org/10.1136/ medhum-2014-010602).

${ }^{1}$ Institute of Social and Cultural Anthropology, University of Oxford, Oxford, UK

${ }^{2}$ Rosie Kay Dance Company, Birmingham, UK

\section{Correspondence to} Dr Karin Eli, Institute of Social and Cultural Anthropology, University of Oxford, 51-53 Banbury Road, Oxford OX2 6PE, UK;

karin.eli@anthro.ox.ac.uk

Accepted 11 December 2014 Published Online First 6 January 2015
CrossMark

To cite: Eli K, Kay R. Med Humanit 2015;41:63-68.

\section{ABSTRACT}

Although narrative-based research has been central to studies of illness experience, the inarticulate, sensory experiences of illness often remain obscured by exclusively verbal or textual inquiry. To foreground the body in our investigation of subjective and intersubjective aspects of eating disorders, we-a medical anthropologist and a contemporary dance choreographer-designed a collaborative project, in which we studied the experiences of women who had eating disorders, through eight weeks of integrating dance practice-based, discussion-based and interviewbased research. Grounded in the participants' own reflections on choreographing, dancing and watching others perform solos about their eating disordered experiences, our analysis examines the types of knowledge the participants used in choreographing their dance works, and the knowledge that they felt the dance enabled them to convey. We find that the participants consistently spoke of feeling as guiding their choreographic processes; they also said the experiences they conveyed through their dance works were centred in feelings, rather than in practices or events. Through dance, the participants said they could communicate experiences that would have remained unspoken otherwise. Yet, notably, dance practice also enabled participants to begin defining and describing their experiences verbally. We suggest, therefore, that through engaging participants in contemporary dance practice, we can begin to identify and address embodied experiences of illness and recovery that may be silenced in speech or writing alone.

\section{INTRODUCTION}

In the medical humanities, narrative has been used widely to explore illness experiences. Through eliciting, recording and interpreting participants' accounts, researchers have examined how people frame causation and course, what themes they deploy in their sense-making and communicative processes, how they perform identity and which lexicons or plotlines loom large. ${ }^{1-4}$ But, as Angela Woods ${ }^{5}$ and others have suggested, ${ }^{6-8}$ while undeniably valuable, illness narratives are limited by their forms and underlying assumptions. Though often cast as essential to the experience of major illness, ${ }^{9} 10$ narrative, argues Woods, is far from universal or 'natural', and the templates it offers leave many experiences unexpressed. ${ }^{5}$

In this project, we aimed to explore the experiential aspects of eating disorders. Our research aim resonated with other work in medical anthropology -work which, in large part, relies on narrative. For logistical and ethical reasons, anthropologists have limited capacity to act as participant observers in the everyday lives of people with eating disorders outside clinical settings. As clinically centred participant observation, by necessity, contextualises embodied experience within institutional strictures and regulations, ${ }^{11-13}$ narrative continues to provide a primary means of addressing the lived experience of eating disorders beyond the clinical realm. ${ }^{14-18}$ Yet our project sought to find a different way of addressing experience; following Csordas' emphasis on 'the body as the existential ground of culture', ${ }^{19}$ we wanted to delve into, and deepen, the understanding of eating-disordered experiences by illuminating the sensory elements they hold. Specifically, we aimed to design a study that would foreground the body, and in which sensory experience and the memories thereof would be expressed first through the body, rather than forced into immediate verbal translation in interview. ${ }^{20}$

We thus turned to contemporary dance as a means of constructing and communicating experiences of eating disorders and recovery. Dance invokes elements of embodied experience central to anthropological inquiry. ${ }^{21}$ With dance being, quite literally, a practice, it involves the shaping and expressing of each dancer's habitus, a habitus moulded through time and technique, somatic possibilities and experiential actualities. ${ }^{22}{ }^{23}$ Dance, moreover, constitutes lived experience in the here and now. As the dancer immerses herself in remembered spaces and times, she embodies these experiences in the moment. ${ }^{24}$ Dance, then, is marked by presence; it unveils embodiment through dialectics of being and creating, imagining and acting, collapsing binary divisions of mind and body, thought and feeling. ${ }^{25}$ And contemporary dance, specifically, enables the exploration of identity, experience, meaning, context, history, the future and the body, as they all intersect in present-tense movement. ${ }^{26}$

In this paper, we aim to identify what kinds of knowledge about the embodiment of eating disorders we can gain through dance, drawing on dance practice-based research we conducted with women who had experienced eating disorders. Rather than relying on our observations and interpretations of the participants' dancing, we follow Drid Williams' argument that the meaning of dance resides in the dancemaker's own experience, ${ }^{27}$ and thus emphasise the participants' own reflections on the experience of choreographing and dancing a solo based on their eating disorder experiences, as well as their accounts of observing other participants perform their solos. Specifically, we ask: What knowledge did the participants employ in choreographing their solos? What knowledge did they feel the dance enabled them to convey? 


\section{METHODS}

\section{Participants and recruitment}

Seven women took part in the study. The participants were aged 19-31 years and were involved in full-time education or employment at the time of the study. While all participants could be described as white, they were of different nationalities (British, German, French and United States). The participants' eatingdisorder experiences were varied; most described experiences that could be categorised as anorexia nervosa (or its subclinical variants), but some described experiences of bulimia nervosa or binge eating disorder. Three of the participants received treatment for their eating disorders, while the other four did not. The participants' duration of eating disorder ranged from approximately one year to over 15 years. All participants identified as recovering from their eating disorders, to varying degrees, with lingering emotional, body image and eating issues. The group had a wide range of dance experience, with four participants who had a strong amateur dance background and three who had no dance training at all.

To recruit participants, we advertised the project through fliers posted locally and through an announcement sent to a local dance society's e-newsletter. KE sent recruitment information via email to one participant with whom she was acquainted, and who had expressed initial interest in the study. The recruitment announcements invited those interested in more information to contact $\mathrm{KE}$ via email. Each person who wrote to $\mathrm{KE}$ received an information sheet about the project. Potential participants who, after reading the information sheet, expressed interest in participating and could commit to attending the full workshop were invited to a $30 \mathrm{~min}$ interview with both researchers. During the recruitment interviews, we introduced ourselves and the project, asked the participants about the general details of their eating disorders and what motivated them to participate in the project, and ascertained whether they had health issues that might prevent them from participating safely or requiring of special attention. In all, we recruited eight participants; one participant left several days before the first session, due to a health issue.

\section{Ethics}

The study was reviewed and approved by the University of Oxford's Central University Research Ethics Committee. All participants were provided with detailed information sheets, and all participants signed consent forms before the workshop sessions began. Pseudonyms are used throughout this article; all identifying details have been concealed or altered to ensure confidentiality.

\section{The workshop sessions and data collection}

For eight consecutive weeks between April and June 2013, we gathered with the seven participants in a non-mirrored black-box studio, for two weekly hours of skill building and creative dance work. The weekly sessions were followed by next-day group discussion sessions, facilitated by both $\mathrm{KE}$ and RK, in which the participants reflected on their experiences of choreographing, dancing, performing and observing others' performances in the studio. The dance sessions were designed to progress from week to week and to develop movement and dance, fitness and coordination, and creative and choreographic skills. Although dance and movement therapy may be used in the treatment of eating disorders, ${ }^{28}$ this study's sessions were aimed at eliciting and facilitating the creative exploration of experience, rather than achieving a therapeutic outcome. Underlying the workshop structure was the idea that the participants were to become choreographers and performers of their own work.
RK led the workshop sessions, and KE acted as participant observer, actively taking part in all skill training and dance assignments (except the final solo work). From week 4 onward, we were joined by RK's teaching assistant, a professional dancer, who took part in dance assignments and served as an additional 'anchor' in the room. We often stayed at the studio after the session with participants who wanted to speak with us. Several participants also stayed in touch with KE by email or telephone following the sessions.

As the workshop's culmination, we asked the participants to choreograph a solo based on their experiences of eating disorder, or related experiences thereof. From week 6 to week 8, as the participants were developing their solos, KE met with each participant individually, for semistructured interviews of 1 $\mathrm{h}-2.5 \mathrm{~h}$ that explored their narratives of illness and recovery and their experiences of creating the solos. The interviews were digitally recorded, as were the discussion group sessions. The participants' solos, and some of the dance works in the other sessions, were video recorded. We made all recordings with the participants' consent. For more about the content of each dance session, see online supplementary appendix 1 .

\section{Data analysis}

In this article, we focus on the participants' accounts of the knowledge they employed in choreographing their solos, and the knowledge they felt the dance enabled them to convey. The analysis, therefore, relies on data collected during the discussion group sessions and the individual interviews with $\mathrm{KE}$, during which we discussed processes of choreographing and watching others perform. Given our interest in the participants' subjective and intersubjective experiences, data were analysed using a phenomenologically informed thematic analysis approach. ${ }^{29}$ The analysis began with familiarisation: KE closely listened to all recordings, identifying segments of salience for this analysis. She then transcribed these segments verbatim, read and reread the collated transcripts several times and made initial codes to denote the participants' interpretations of their experiences (eg, 'dance corrects misperceptions', 'eating disorders necessitate different sense-making'). Based on the initial codes, KE reanalysed the entire corpus for subthemes. The subthemes were then brought together to form the themes reported in this article; this process was supported by a quote-based mapping of the themes, in which all relevant participant quotes were labelled and thematically grouped. RK reviewed the themes, and the authors discussed the analysis, examining and resolving disagreements and reaching consensus on the reporting of data.

\section{RESULTS AND DISCUSSION Working with/from feeling}

In their solo brief, the participants were asked to create a work that expressed their experience of eating disorder. We asked them to draw on the skills they developed in the first five sessions, skills that enabled them to explore movement through body parts, space, relationships and the emotions.

Yet, while the participants were not asked directly to choreograph their solos with or from feelings, the centrality of feeling occurred, time and again, in their interviews. As Lauren described it, in choreographing her dance, she allowed herself to feel, or 'connect with', painful memories which, she told KE, she had numbed, over the years, through anorexia.

Having to express something in dance made me-I was forced to think about stuff I don't normally think about.... I mean, I've, I've done quite a lot of therapy, so I've done quite a lot of the 
talking thing... but I think I connected with stuff in a different way, I, I actually felt stuff in myself inside me, that you can think about, but it feels different when you go back to that place and you feel that stuff again. (Lauren)

Lauren's dance itself, she said, emanated from a strong feeling she had one morning - a feeling she knew was related to the past-which she immediately described in writing, and later, sought to capture in movement. This exploration was, at times, difficult; Lauren said that when she performed part of her solo to the group, she was suddenly overcome by a feeling of 'real fear', which she later understood was related to the traumatic recollections she explored through the dance. But this working with feeling, she said, did not feel 'unsafe', in her words, only different.

For Gia, who, like Lauren, had eating disorders for over a decade, working with feeling entailed a conscious process of recollecting and evoking sensation in her body.

I've gotten into a space where I feel comfortable reliving my experience, my eating disorder in my head, and trying to even go from the head to, to you know, seep into my body, so that it becomes a physical feeling, not just mental... (Gia)

Gia said she chose to recollect, embody and explore five periods of her life in her solo dance. The progression of these periods was linear, but she did not conceptualise them as a narrative of 'what happened'. Instead, Gia's focus was on her own changing sensations and perceptions, from one period to the next, and how they came (back) into being, in her body, through the dance.

Feeling was what provided a starting point and choreographic focus for the creation of the solo; it was also the focus of the choreographic exploration itself. For Jane, the distinction between the two was crucial. Jane said she had found it difficult, even impossible, to work from feeling-she described the experience of connecting with emotions as 'paralysing'. Yet feeling was at the centre of her work. As Jane explained it, while she created her solo through reminiscences of feeling, which she kept safely at bay during the creative process, she wanted her solo to explore the very core of the feelings that underlay her eating disorder.

That's really something that I wanted to address in my dance, that kind of, sense of powerlessness, inactivity masked by routine, masked by routine movements like walking into the supermarket.... I want to capture that sense of emptiness, of a void within you that you need to fill, of a kind of gloominess, like an ache, but that also is so, kind of deep that it makes you feel like you're not there, like you don't exist, like you're not a being, you lose your sense of agency into it... this kind of, a void of darkness that you create within yourself, umm, and the loneliness, and also the way in which you push other people away... (Jane)

Jane's solo had little plot; while she told KE that the progression of movement was meant to convey a transition in her own life-moving past the eating disorder-the solo's focus was on conveying embodied experience, unbound by time and narrative. Like Lauren, Gia and the other participants, Jane was concerned with creating a feeling-centred body of knowledge on her eating-disordered experience-one which provided a tangible alternative to plot-driven narrative, and allowed for an embodied, rather than discursive, sense-making.

\section{Transcending templates}

When I started to think about, okay, how I'm going to do this, and this thing that I do have all these blanks, but I also have these very specific experiences and that I started noting them down and how I could express them, and that made me then start feeling like I wanted to be able to put all of them in and be able to tell about, I mean tell in the dance, about all these specific memories and experiences. (Eve)

Eve's 'blanks'-extensive periods of her eating disorder which she could not remember-were a central part of her experience. Eve mentioned these 'blanks' at various stages of the project, as early as the recruitment interview phase; she was bothered by her inability to remember the entire arc of her disorder, by the 'leaps' her memory made from one episode to another-leaps that made her feel as if she could not narrate what had happened to her. The dance, however, enabled her to 'tell' her experience beyond the template offered by narrative structure: to make a whole work-with no apology for blank spaces in her story. Eve and RK discussed how the blanks themselves could form material for her solo. RK told Eve how she had used the concept of purgatory, limbo or nothingness, to create a section in one of her dance works, and they discussed how the physicality of 'stretched time' could feel or look. Following that discussion, RK noted in her journal that Eve began to create her solo in the studio with more energy. On watching Eve's work-in-progress sharing of her solo, RK wrote:

Eve's performance was electric and ghostly-the absences like tension, palpable in her performance.

Unlike Eve, Joyce had remembered nearly the entirety of her eating disorder and had structured her dance in linear format. Joyce, however, said that the experience-focused quality of the dance allowed her to overcome a template that used to guide her narrative-the definition of what an eating disorder is.

I think in the few times where I've described it explicitly... what I end up falling into is like, trying to describe my own, my very personal, like experience or personal reasons... in these sort of terms that would fall under the commonly like expected idea [of] eating disorders.... Whereas with dance, it's like you, it's very difficult to fall into that, feeling you need to describe it in a certain way, or describe it like how other people describe it, because it's like-I have no way of mimicking the way that you move.... It becomes very original. (Joyce)

Like three other participants, Joyce never received professional treatment for her eating disorder. She did not have an official diagnosis, and described wondering, in the past, whether she indeed had an eating disorder, as it did not match her expectations of what anorexia or bulimia might or should be. For Joyce, the process of creating and performing her own dance, as well as watching the other participants' solos, was validating, as she explained: 'there aren't, like, just two categories of eating disorder'. Through dance, Joyce said, she could both express and observe the nuances of individual experience; there was no need to align her own eating disorder with textbook expectations so as to 'qualify' for the 'title'.

For Lauren, who had extensive contact with the medical system, the templates of what constituted eating disorders extended beyond the issue of validity. While she was clinically recognised as anorexic, Lauren felt that the supposedly objective measures of her eating disorder-her body weight, her body mass index (BMI), her food intake-were privileged beyond her own experience of the disorder, to the extent that they stood for her eating disorder itself.

In the medical or the psychological world it's all about what your weight was or what your BMI was and-how much you are or aren't eating, or what you're doing with food. But that's not really what having an eating disorder is about-it's about those 
really strong kind of struggles and self loathing and, umm, just struggling to get through that people were expressing through the dance. (Lauren)

Through dance, Lauren said she was able to address those aspects of her experience which were often obscured, even negated, by the templates provided in the medical system. The dance, she said, allowed her to capture the ambiguities of her eating disorder-the pain and the longing, the suffering and the desire, and what it means to live through and, with it, in ways she could not express within the ready-made templates of food, calories and weight.

\section{Narrative logic versus 'bodily logic'}

All participants, including those who had never danced before, spoke of a 'logic' of movement that allowed them to convey their experience. In this excerpt, from a discussion we held with the group following the first solo-work session, Alicia and Gia brought up the word 'logic' to contrast telling and dancing their eating disorders:

Alicia: I think, because it's not a very logical thing, it's a good thing to express in dance. Because if you say, if you kind of put a factual spin on it, it doesn't make much sense. (...)

Gia: and you've got that horrendous thing where, as you say, as soon as you're telling the story you necessitate, it necessitates a logic of storytelling, so it's like, well why did you? Like, I don't know.

For Alicia, dance offered a medium through which to express the illogic of eating disorders. Although the solo did require some structure and choreographic sense-making, the format, as she explained it, was open-ended enough to allow for the expression of experience that might otherwise need to be clarified and made cogent in speech. And, as Gia explained, the 'logic of storytelling' in speech also required a sense-making that perhaps she was not ready to do; dance allowed her to articulate eating-disordered experiences in feeling and motion, yet keep them raw and unencumbered by explanations.

In finding the internal logic of their solos, the participants also had to come to terms with the logic of their own bodies. The links and disconnects between imagined and actualised movement came into the foreground during the solo-work sessions. For Gia, this was the emergence of a 'bodily logic':

You have an idea of what you want to do, and you do it, and your body is just kind of doing something else... So in that sense, the logic-the intellectual, or the, the conscious logic, kind of gets a bit split, and then you've got this very bodily logic that kicks in. (Gia)

Gia later described feeling positively surprised by the movement she found in her body-by the embodied actuality of dancing the solo she had planned.

While it might have presented a constraint on the range and style of motion each participant could embody, working with the body's own logic was part of the creative engagement.

I kind of feel what I want to do, but I can't to do that, so I'm up against that barrier some of the time, so trying to find ways around that, try and stay true to that feeling, to what I want to express... (Lauren)

Lauren identified 'staying true to that feeling' as the core of her solo project, and later told KE she thought of creative uses of the body and space, based on the skills sessions, to capture the feeling she wanted to evoke with motion. The logic she found in her body sharpened both concept and practice.

\section{Conveying the unspeakable}

One of the strengths of creating dance, as expressed by the participants, was in enabling the expression of those aspects of the eating-disordered experience which would have otherwise remained unarticulated. The unspeakable moments of eating disorder were related not to events, but to sensations-sensations which were part of eating-disordered embodiment, and which the participants felt were inaccessible to non-eating-disordered others, and inexpressible through language. Reflecting on the group discussion concerning narrative versus dance, Catherine said:

There are some things which, like, I just have never been able to find words for, like I can't ever explain what, like, a panic attack feels like in words, and I find that quite difficult, and especially because the last two years I found myself sitting in a lot of doctors' offices....I've always found it quite difficult to express how it actually felt or what my actual experience-like, I can tell people, oh, it started here, umm, and I went to treatment here, and my weight was this, and stuff like that, but that's all kind of like stuff that has words attached to it already and that's just fact, it doesn't actually express what it felt like or what my experience of it was like or how I saw it or anything like that. (Catherine)

Identifying the easily narrated aspects of her eating disorder as 'stuff that has words attached to it already', Catherine alluded to the sense of unoriginality and collectivity that these signposts of illness conveyed. The realities that inhered in her body-in experiences such as having a panic attack-seemed unbridgeable by language; there were no experience-near words that could allow her a transition from feeling to narrative, to reach out and express her sensory world to a listener.

Later, Catherine added that she knew some people were more comfortable with speech, while others were with movement, and that she belonged to the latter group. But feeling comfortable with speaking did not make all aspects of experience expressible. Gia, who said she told 'everyone' about her eating disorder-including family, friends, colleagues and acquaintances-explained to the group that while she had told her story many times, she found the dance had captured crucial elements of her experience which she could not convey through speech:

I find it also more powerful than talking-I don't know if any of you have that experience. Because I've talked about my eating disorder, and heard other people speaking about their eating disorders, and there's a bit of a casualness about it. Whereas in dance you kind of have this full force of the reality of what would have been, could be, or is. I find that very, very disrupting to my sense of calm, and at the same time, I guess umm, one of the rare moments where I can actually be genuine or authentic. (Gia)

The 'reality' of which Gia spoke contrasted with what she later termed the 'banality' of her eating disorder narrative. Gia said she was emotionally controlled whenever she spoke about her eating disorder: 'I tell it like a story'. This control allowed Gia to be open about her eating disorder, while protecting herself from vulnerability. And yet, these instances of speaking about her disorder lacked the 'authenticity' which she located in feeling. Through dancing, however, Gia said she could both feel and express the lived experience of her eating disorder, which, for her, constituted her 'genuine' reality and 'genuine' self, otherwise obscured by words.

While the participants sought authenticity, they were also mindful of an audience; to express the unspeakable, individual 
experience had to be translated into evocative, if still abstract, movement. When KE spoke with Eve about her process of choreographing the solo, Eve said,

I noticed that in those early stages something that kept popping up in my head was that I thought, oh, but that doesn't express the pain strongly enough....[it] seems to me to again be this kind of thing of wanting people to-see and notice and understand...(Eve)

For Eve, her duty as both dancer and sufferer was not only to capture authentic feeling, but to communicate it. This communication entailed the choreographing of movement to elicit visceral empathy - an embodied understanding of the pain which, throughout the years, she had never been able to speak of, and for which she had never received help or recognition. And as she choreographed the dance, she sought to convey the fact of pain and its intensity, expressing that which is inherently unspeakable.

\section{Visceral recognition, embodied connection}

The participants held multiple roles in this project-choreographer, dancer, performer and observer. When asked about their experiences of watching the other group members perform their solos, all participants spoke of feeling a strong sense of connection. The experience went beyond what each of the dances 'told'; as Alicia explained, identification inhered in the movements themselves.

They're very different, but there's bits I can relate to in all of them and sort of, not feel like, oh, I know what they're representing, but just-I know that feeling. Like, there's a lot of people touching their skin in lots of them, which is something I do a lot, even now, like, it's just that constant checking your body and checking it's alright. (Alicia)

Like the other participants, Alicia did not try to guess at the individual stories that underlay each of the solos. Indeed, the participants were not exposed to one another's narratives-we did not discuss individual experiences of eating disorder with the group, only in the one-on-one interviews KE held with each of the participants. The sense of connection was one that transcended similarities in narrative; it inhered in a tacit recognition of shared sensory experience, a recognition related, in motion, from dancer to observer. These moments of recognition were described by another participant, Eve, as knowing she was 'in the right place' and feeling she was not alone.

While many participants cited similarities in movement motifs, the sense of embodied connection did not depend on direct likeness:

Sometimes I saw something of like, myself, something I related to in someone else's dance, and I was like, I never would have even expressed it that way or I didn't know how to express that. And then I see you do that, and I'm like, yeah, that's it, that's right. (Catherine)

The ineffable sense of 'that's right' which Catherine described, was one invoked by movement that could have been one's own, and yet (crucially) was not. The participants spoke of the empathy they felt for one another as they performed, an empathy that went beyond compassion to encompass a sense of the other's experience. 'Seeing oneself in others' meant more than seeing a reflection of oneself; it meant recognising a range of movements, and movement possibilities, through one's own embodied being.

\section{CONCLUSION}

The participants described dance as a medium through which they could communicate otherwise unspoken experience to others, as well as viscerally recognise and empathise with another's experience. Feeling was at the heart of both the creative process, as the participants actively invoked and engaged with embodied memories, and the dance performances themselves, as each participant conveyed her eating-disordered experience through viscerally evocative movements, and as those watching said they could recognise a dancer's experience through their own bodies.

The participants used their solo choreographies to express their eating-disordered experiences as feeling-centred. It was feeling which they identified as the crux of experience-that which constituted their eating disordered realities and authentic selves. Notably, while the participants could state feeling in wordsindeed, they spoke of feelings in the interviews and group discussions-they also conveyed that the intensity, sensory qualities, ambiguities and complexities of feeling eluded words. ${ }^{30}$ Through the dance, then, the participants engaged with the unspeakable, developing a feeling-centred body of knowledge.

We acknowledge that many of the critiques levelled against narrative can be applied to dance. While dance is based in embodied being and expression, it is neither a pan-human expressive form, nor is it 'natural': the body is always-already encultured. ${ }^{31}$ Dance is also subject to structure; while danced expression may rely on tacit sense-making, rather than on preestablished vocabularies, it is made intelligible through communicative modes embedded in the cultures from and within which it is created and performed. ${ }^{32}{ }^{33}$ Moreover, choreography entails the synthesising and stylising of experience. The participants did not convey experience in its inchoate immediacy; rather, in creating a dance, they reflected, selected and strove to make sense to themselves and to others.

We would like to suggest, however, that dance can change the emphasis of storytelling in healthcare contexts. The participants' solos were not accounts of 'what happened', but rather danced renderings of 'how it felt'. Their creating of, and reflections on, feeling-centred bodies of knowledge opened up possibilities for further inquiry into their experiences of eating disorders. As this paper showed, dance was not only a means of conveying the unspeakable, but was also a platform that allowed the participants to begin defining and discussing elements of experience they found difficult to capture in speech alone. The process of solo development, in particular, encouraged reflective verbal expression of the sensory dimensions of eating disorder experiences. Through posing questions about illness experience whose answers were both danced and spoken, we were able to acknowledge multiple beginnings and endings, the variety of possible tellings in form and time (episodic, expressive, linear, circular), and those feelings and embodied memories which might have otherwise remained unspoken. Thus, we suggest that, in bringing the sensate body to the foreground of analysis, dance allows us to address lived experiences of illness and recovery.

Acknowledgements We thank the women who participated in this project for generously sharing their experiences, reflections and choreographies. We also thank our project's Pl, Stanley Ulijaszek.

Contributors KE and RK designed the study; KE conducted the analysis and drafted the manuscript; KE and RK revised the work critically; KE and RK provided final approval of the version to be published; KE and RK agree to be accountable for all aspects of the work in ensuring that questions related to the accuracy or integrity of any part of the work are appropriately investigated and resolved.

Competing interests None.

Ethics approval University of Oxford's Central University Research Ethics Committee.

Provenance and peer review Not commissioned; externally peer reviewed. 


\section{REFERENCES}

1 Good BJ, Del Vecchio Good MJ, Togan I, et al. In the subjenctive mode: epilepsy narratives in Turkey. Soc Sci Med 1994;38:835-42.

2 Skultans V. Theorizing Latvian lives: the quest for identity. J Roy Anthrop Inst 1997;3:761-80.

3 Riessman CK. Strategic uses of narrative in the presentation of self and illness: a research note. Soc Sci Med 1990;30:1195-200.

4 Mattingly C. Healing dramas and clinical plots: the narrative structure of experience. Cambridge: Cambridge University Press, 1998.

5 Woods A. The limits of narrative: provocations for the medical humanities. Med Hum 2011;37:73-8.

6 Atkinson P. Narrative turn or blind alley?. Qual Health Res 1997;7:325-44.

7 Bury M. IIIness narratives: fact or fiction?. Sociol Health IIIn 2001;23:263-85.

8 Charmaz K. Stories and silences: disclosures and self in chronic illness. Qual Inq 2002;8:302-28.

9 Frank AW. The wounded storyteller: body, illness, and ethics. London: University of Chicago Press, 1995.

10 Charon R. Narrative medicine: a model for empathy, reflection, profession, and trust. J Am Med Assoc 2001;286:1897-902.

11 Gremillion H. Feeding anorexia: gender and power at a treatment center. London: Duke University Press, 2003.

12 Warin M. Transformations of intimacy and sociality in anorexia: bedrooms in public institutions. Body Soc 2005;11:97-113.

13 Lester RJ. Critical therapeutics: cultural politics and clinical reality in two eating disorder treatment centers. Med Anthropol Q 2007;21:369-87.

14 Garrett C. Beyond anorexia: narrative, spirituality and recovery. Cambridge: Cambridge University Press, 1998.

15 Shohet M. Narrating anorexia: "full" and "struggling" genres of recovery. Ethos 2007;35:344-82.

16 O'Connor RA, Van Esterik P. De-medicalizing anorexia: a new cultural brokering Anthropol Today 2008;24:6-9.
17 Cheney AM. Emotional distress and disordered eating practices among southern Italian women. Qual Health Res 2012;22:1247-59.

18 Lavis A. The substance of absence: exploring eating and anorexia. In: Abbotts E-J, Lavis A, eds. Why we eat, how we eat: contemporary encounters between food and bodies. Aldershot: Ashgate, 2013:35-52.

19 Csordas TJ. Embodiment as a Paradigm for Anthropology. Ethos 1990;18:5-47.

20 Carel H. Phenomenology as a resource for patients. J Med Philos 2012;37:96-113.

21 Farnell B. Moving bodies, acting selves. Annu Rev Anthropol 1999:28:341-73.

22 Bourdieu P. Outline of a theory of practice. Cambridge: Cambridge University press, 1977.

23 Desmond JC. Embodying difference: issues in dance and cultural studies. Cult Critique 1993;26:33-63.

24 Sklar D. Reprise: on dance ethnography. Dance Res J 2000;32:70-7.

25 Fraleigh SH. Dance and the lived body: a descriptive aesthetics. Pittsburgh: University of Pittsburgh Press, 1987.

26 Albright AC. Choreographing difference: the body and identity in contemporary dance. Middletown: Wesleyan University Press, 2010.

27 Williams D. Anthropology and the dance: ten lectures. Urbana: University of Illinois Press, 2004.

28 Frisch MJ, Franko DL, Herzog DB. Arts-based therapies in the treatment of eating disorders. Eat Disord 2006;14:131-42.

29 Braun V, Clarke V. Using thematic analysis in psychology. Qual Res Psychol 2006;3:77-101.

30 Wikan U. Beyond the words: the power of resonance. Am Ethnol 1992;19:460-82.

31 Krieger N. Embodiment: a conceptual glossary for epidemiology. J Epidemiol Community Health 2005:59:350-5.

32 Kaeppler AL. Dance ethnology and the anthropology of dance. Dance Res J 2000;32:116-25.

33 Kringelbach HN, Skinner J. The movement of dancing cultures. In: Kringelbach HN, Skinner J, eds. Dancing cultures: globalization, tourism and identity in the anthropology of dance. Oxford: Berghahn Books, 2012:1-28. 